\title{
Galaxy Rotation in the Space of Four Distance Dimensions
}

\author{
Ahti Rahikainen \\ Merkkipuuntie 2 L 39, Vantaa, Finland \\ Email: ahrahik.zz@kolumbus.fi
}

How to cite this paper: Rahikainen, A (2020) Galaxy Rotation in the Space of Four Distance Dimensions. World Journal of Mechanics, 10, 83-94.

https://doi.org/10.4236/wjm.2020.107007

Received: June 1, 2020

Accepted: July 18, 2020

Published: July 21, 2020

Copyright $\odot 2020$ by author(s) and Scientific Research Publishing Inc. This work is licensed under the Creative Commons Attribution International License (CC BY 4.0).

http://creativecommons.org/licenses/by/4.0/

\begin{abstract}
This study contains the solution of the dark matter mystery of spiral galaxies by using the space of four distance dimensions $x, y, z, x^{\prime}$, in which $x^{\prime}$ is the fourth distance dimension. The calculation of galaxy rotation has been presented in the space of four dimensions by using two dimensional section $x, x^{\prime}$ and three dimensional section $x, y, x^{\prime}$. The four dimensional mass $M$ which generates the main gravitation field of galaxy is located at the fourth dimension at the distance $x^{\prime}=X^{\prime}$ and other dimensions are zero $x=0, y=0$, $z=0$. The method to calculate the speed distribution curve of four dimensional mass $V_{M}$ : the speed distribution curve $V_{M}$ is calculated by using the equation in which the gravitational force is equal to the centrifugal force of rotation. The solution of this equation yields the speed distribution component $V_{M}$ of the four dimensional mass $M$ and the value of the mass $M$. In the publication [1] has been presented rotational speed distributions curves of the galaxy NGC 3198. The speed distribution curve of galactic halo in that publication corresponds to the speed distribution curve $V_{M}$ of four dimensional mass $M$ of this study. In order to find out how well this four dimensional model functions, the speed distribution curve $V_{M}$ of four dimensional mass $M$ has been calculated by using two pairs of rotational radius and speed values. The conclusions and findings: the calculated distribution curve $V_{M}$ was a good match for the halo curve of the publication [1]. Furthermore, four rotational speed distribution curve $V_{M}$ was calculated using different values of the distance $X^{\prime}$, which yielded different values for the maximum radius of galaxy. In this manner the different galaxy models of the publication [2] were obtained. By that means the solution of dark matter mystery has been proved.
\end{abstract}

\section{Keywords}

Galaxy Rotation, Dark Matter, Atom Theory 


\section{Introduction}

The Sun and other stars which are visible in the sky are a part of the Milky Way galaxy. The Milky Way is a typical spiral galaxy which consists of a thin rotating disk of stars, and in its middle is a bulge of dense located stars. The stars of the Milky Way rotate round about the center of galaxy at a certain rotational speed. The Sun and the earth are located in the middle of the disk of the Milky Way at the distance of 25,000 light years from its center, and they rotate round the center at about the speed of $220 \mathrm{~km} / \mathrm{s}$. Edwin Hubble was the first astronomer who inferred that spiral nebulae are galaxies which are located at great distances from the Milky Way, publication 1929. In publication [3] 1978 it was found that masses of galaxies were underestimated by a factor of 10 or more, and therefore also the density of the universe was underestimated by the same factor. The speeds of stars in the galaxies have been measured from the redshifts of the light that they emit. The speed of star results in a change of wave length, which is called redshift. In universe, all stars at great distances have the change of wave length towards red, and therefore it has been inferred that the universe is expanding. By using the redshifts Vera Rubin did measurements of the rotating speeds of galaxies, and she found that the stars rotate in the outer part of galaxy approximately at the same speed, publication [2] 1983. The speed distribution is at the center of galaxy zero, after that it rapidly increases until at a certain value it remains constant. The matter of spiral galaxy is concentrated as a great dense bulge at the center of galaxy, and the rest of galactic matter forms a large rotating spiral disk. The gravity force of this kind of galactic matter distribution reduces approximately with inverse of square of distance from the center of galaxy. Therefore the constant value speed distribution is not resulted only from the visible and dark matter of the center bulge and disk of the galaxy. There must be a great amount of some other kind of dark matter effecting to the rotation of stars. What kind of mass distribution will make the constant rotational speed distribution to the outer part of galactic disk. It should be probably a ball shape dark matter formation. It is inferred that the missing dark matter can be even $90 \%$ of the total mass of galaxy, and it would probably be planet like heavenly bodies, gas and dust clouds concentrated as a huge ball or halo round the disk of stars. This kind of galactic structure may possibly realize the redshift distribution. It was supposed to be like that, and accordingly that kind of heavenly bodies round the galactic disk were search for. The super accurate images of space telescope Hubble made it possible to observe dim objects at great distances. However, the results of observations did not meet the expectations. Sufficient amount of dark matter above the galactic disk was not found. The measurements of the space telescope Hubble have not given support to this theory, the publication [4] 1995. It seems that dark matter in the halo of galaxy is not that massive that it can explain the constant speed distribution. The search for dark matter halo continues in publications [5] 2010 and [6] 2012. After that, the search for dark matter has been concentrated in the first place to fundamental particles. It must be such funda- 
mental particle which has mass and force of gravity. The particle must not have other forces, because otherwise it would have been observed already. Fundamental particles like this have been searched in mine shafts in which there are disturbing factors as little as possible. Richard Panek has written on the latest difficulties in the research of the universe, publication [7] 2020. Astronomers have calculated how fast the universe is expanding. Two different calculation methods have two different results. The cause of the discrepancy may be the fact that physics has new unknown phenomena. In this study, it has been developed the idea that dark matter is located at fourth distance dimension above the center of galaxy. In the same manner as a structure of three dimensions can be drawn in the paper of two dimensions, a structure of four dimensions can also be drawn in the paper of two dimensions. Therefore the determination of the location of dark matter in fourth dimension is no problem.

\section{Method of Calculation}

In the following, a simple method has been presented which makes possible to see the space of four distance dimensions, and to do calculations in it. In Figure 1 , a box is drawn in three dimensional space $x, y, z$. In Figure 2, the same box is drawn in two dimensional space $z, x$ and in Figure 3, it is drawn in two dimensional space $z, y$. There is still third possible coordination $x, y$, but it is not needed to determine the shape of the box. Here it is seen that a structure of three dimensions can be drawn in coordination of two dimensions. In the same manner a structure of four dimensions can be drawn in coordination of three dimensions $x, y, z, \quad y, z, x^{\prime}, \quad x, z, x^{\prime}, \quad x, y, x^{\prime}$. If the structure is simple, it is possible that only one two dimensional coordination is needed to determine the form of structure.

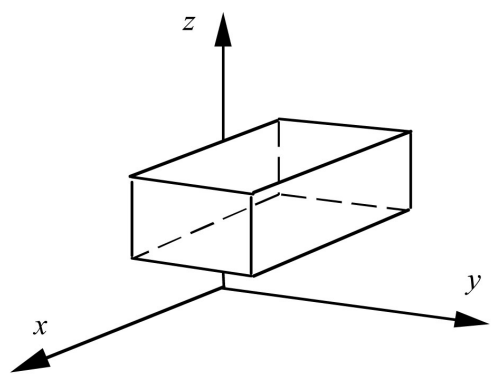

Figure 1. A box drawn in coordination of three dimensional space $x, y, z$.

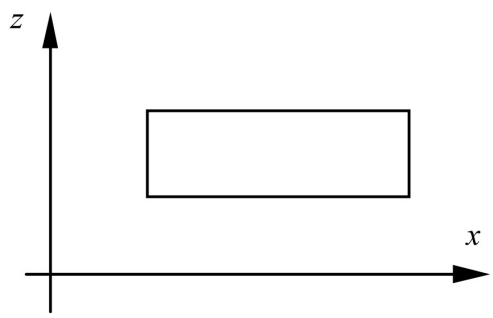

Figure 2. The box of Figure 1 drawn in coordination of two dimensional space $Z, X$. 


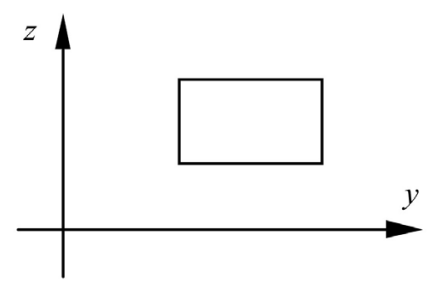

Figure 3. The box of Figure 1 drawn in coordination of two dimensional space $z, y$.

In Figure 4 the four dimensional space $x, y, Z, x^{\prime}$ is presented as a drawing of two dimensional coordination system $x, x^{\prime}$. The universe is comprised of three dimensions $x, y, z$ and the enlarged universe has four dimensions $x, y, z, x^{\prime}$. The coordination system of Figure 4 is constructed from the coordination system of Figure 1 so that the galaxy rotates at the plane of $x, y$ coordinates and the coordinate $z$ has been exchanged to the coordinate of the fourth dimension $x^{\prime}$. In the figure a star of mass $m$ rotates round about the center of galaxy $O$. The rotation axis of the galaxy is the coordinate $x^{\prime}$ and the center of gravity of four dimensional mass $M$ is at the distance $X^{\prime}$ from the center of galaxy $O$. The mass of the star $m$ and mass concentration $M$ draws each other with the force of gravity. Because the three dimensional gravity force of the star is lacking the force of fourth dimension, the force that has effect on the star is $F \cos \alpha$.

It seems that this model fits very well for the measurements. Near the center of galaxy, rotational speeds from the redshift measurements and theoretically calculated speeds from the light intensities of rotating stars have relatively good correspondence. In the center of galaxy, the gravity force between the star mass $m$ and the four dimensional mass concentration $M$ is in the $x^{\prime}$ axis direction, and it disappears completely. At the border areas of the galactic rotating disk the gravity force component in the $x$ axis direction $F \cos \alpha$ increases which is according to the redshift measurements. Even over $90 \%$ of the gravity forces acting at the border areas of galaxy cannot be explained by the masses of the visible stars. This model of the space of four dimensions can explain the weird rotation speeds of galaxies.

\section{Numerical Calculations of the Rotational Speed of Galaxy}

The mystery of dark matter in the rotation of galaxies is a common subject matter in the study books of cosmology, publication [8]. In this paper the function of fourth distance dimension has been studied in that mystery. In Figure 5 a star with mass $m$ rotates round about the center of galaxy along the circle of radius $R$. As the star rotates round about the center of galaxy, the force of gravity effecting on the star is equal to the centrifugal force of its rotation movement.

In the following star's rotational speed $v$ round the center of galaxy has been derived. The speed components of star's rotational speed $v$. speed component from visible matter $V_{n}$, ordinary dark matter $v_{p}$, four dimensional mass $M V_{M}$. The differentia of the gravitational force is equal to the differentia of the centrifugal force. 


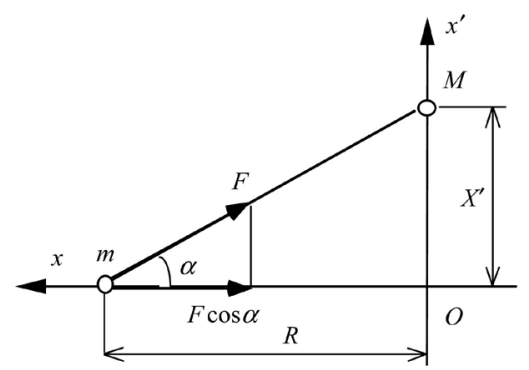

Figure 4. A star mass $m$ rotates round about the center of galaxy $O$ at the distance $R$. Fourth dimensional mass $M$ is located at the distance $X^{\prime}$ from the center of galaxy.

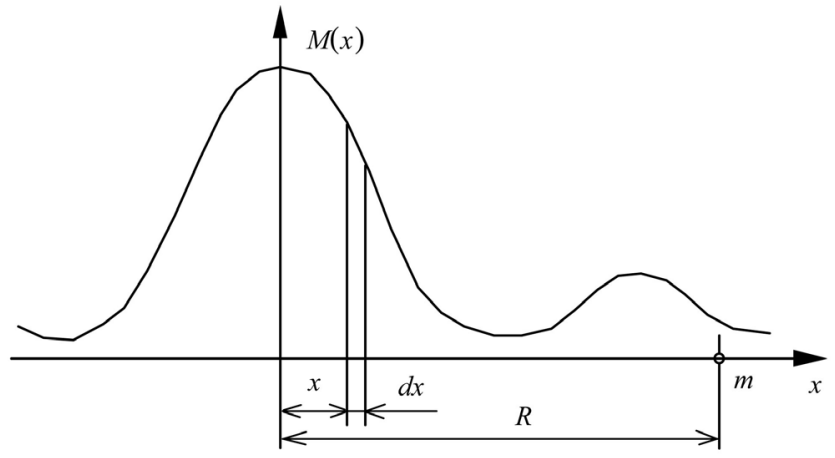

Figure 5. Calculation of the effect of visible galactic mass $\Delta M(x)=M(x+\mathrm{d} x / 2) \mathrm{d} x$ on the rotational speed component $v_{n}$ of the star which mass is $m$.

$$
\frac{\gamma m \Delta M(x)}{(R-x)^{2}}=\frac{m}{R} \mathrm{~d}\left(v_{n}^{2}\right)
$$

Summation yields the following equations.

$$
\gamma \sum m \frac{\Delta M(x)}{(R-x)^{2}}=m \int \mathrm{d}\left(v_{n}^{2}\right)=\frac{m v_{n}^{2}}{R}
$$

The centrifugal force components are from visible mass, ordinary dark mass and four dimensional mass $M$.

$$
\frac{m v_{n}^{2}}{R}, \frac{m v_{p}^{2}}{R}, \frac{m v_{M}^{2}}{R}
$$

The following equation is obtained by adding the three centrifugal force component.

$$
\frac{m v^{2}}{R}=\frac{m v_{n}^{2}}{R}+\frac{m v_{p}^{2}}{R}+\frac{m v_{M}^{2}}{R}
$$

or

$$
v^{2}=v_{n}^{2}+v_{p}^{2}+v_{M}^{2}
$$

Total rotational speed curve $v$ and rotational speed curve of visible mass $V_{n}$ are known, rotational speed curve of ordinary dark matter $v_{p}$ is not knows, but it can be concluded that it is the same form as the speed curve of visible matter. The rotational speed curve $V_{M}$ of the four dimensional mass $M$ is not known and it 
will be calculated by using the knowledge of the rotational system. In Figure 6 a star which mass is $m$ rotates round about center of galaxy $O$ at distance $R$. Center of gravity of the four dimensional mass $M$ is on the rotation axis $x^{\prime}$ at the distance $X^{\prime}$ from the center of galaxy. The galaxy has two rotational axes $z$ and $x^{\prime}$. This can be seen as follows: The rotating star system in four dimensional space $X$, $y, z, x^{\prime}$ in Figure 6 is converted to the system of oscillating atom about zero point $O$ in three dimensional system $x, y, x^{\prime}$. If the oscillating atom is at the point of mass $m$, and it is oscillating along axis $x$ about point $O$, it has two axes about which it oscillates $y, x^{\prime}$ in three dimensional space $x, y, x^{\prime}$. In the same manner the star rotating round about point $O$ has two axes $z, x^{\prime}$ round about which it rotates in four dimensional space $x, y, z, x^{\prime}$. The gravity force of three dimensional mass decreases in inverse value of square of distance, and it can be supposed that the gravity force of four dimensional mass decreases in inverse of cube of distance, and therefore the gravity force of four dimensional mass $M$ is supposed to decrease at third power of distance, and it is in Figure 6

$$
F=\frac{\gamma m M}{\left(R^{2}+\left(X^{\prime}\right)^{2}\right)^{3 / 2}}
$$

In which the component in the direction of $x$-axis is

$$
F \cos \alpha=\frac{R \gamma m M}{\left(R^{2}+\left(X^{\prime}\right)^{2}\right)^{2}}
$$

The centrifugal force component corresponding to the four dimensional mass $M$ is

$$
\frac{m v_{M}^{2}}{R}
$$

Calculation of the speed distribution curve of the four dimensional mass $M$. The gravitational force is equal to the centrifugal force.

$$
\frac{m v_{M}^{2}}{R}=\frac{R \gamma m M}{\left(R^{2}+\left(X^{\prime}\right)^{2}\right)^{2}}
$$

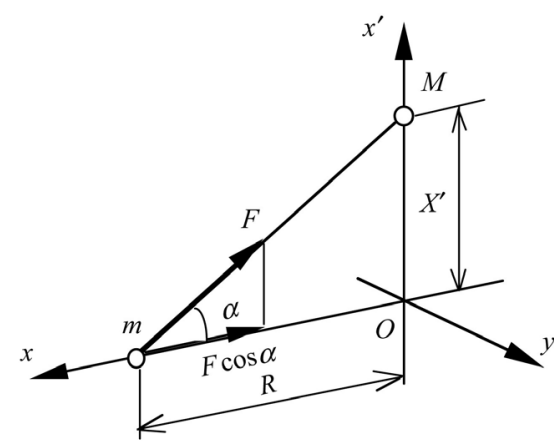

Figure 6. A star of mass $m$ rotates round about the center of galaxy $O$ along a circle trajectory of radius $R$. The gravity effect on the star from the four dimensional mass $M$ is in the direction of $x$ axis $F \cos \alpha$. 
The speed component of the four dimensional mass $M$ effecting on the star and the mass $M$

$$
\begin{aligned}
& v_{M}=\sqrt{\frac{R^{2} \gamma M}{\left(R^{2}+\left(X^{\prime}\right)^{2}\right)^{2}}} \\
& M=\frac{v_{M}^{2}\left(R^{2}+\left(X^{\prime}\right)^{2}\right)^{2}}{R^{2} \gamma}
\end{aligned}
$$

Equation (9) at distances $R_{1}$ and $R_{2}$ from the center of galaxy

$$
\begin{gathered}
\frac{m_{1} v_{M 1}^{2}}{R_{1}}=\frac{R_{1} \gamma m_{1} M}{\left(R_{1}^{2}+\left(X^{\prime}\right)^{2}\right)^{2}} \\
\frac{m_{2} v_{M 2}^{2}}{R_{2}}=\frac{R_{2} \gamma m_{2} M}{\left(R_{2}^{2}+\left(X^{\prime}\right)^{2}\right)^{2}}
\end{gathered}
$$

By dividing the two equations above

$$
\begin{gathered}
\frac{R_{2}^{2} v_{M 1}^{2}}{R_{1}^{2} v_{M 2}^{2}}=\frac{\left(R_{2}^{2}+\left(X^{\prime}\right)^{2}\right)^{2}}{\left(R_{1}^{2}+\left(X^{\prime}\right)^{2}\right)^{2}} \\
a=\frac{v_{M 1} R_{2}}{v_{M 2} R_{1}} \\
a\left(R_{1}^{2}+\left(X^{\prime}\right)^{2}\right)=R_{2}^{2}+\left(X^{\prime}\right)^{2}
\end{gathered}
$$

Distance of the four dimensional matter $M$ from the center of galaxy

$$
X^{\prime}=\sqrt{\frac{R_{2}^{2}-a R_{1}^{2}}{a-1}}
$$

Values of total rotational speed distributions $v$ have been calculated from redshifts of rotating stars of galaxies. Rotational speed distribution component of visible stars $V_{n}$ has been calculated from the gravity force of their mass, which has been calculated according to their light intensity, the result of which has been like the distribution curves in Figure 7. It can be supposed that the speed distribution curve from the ordinary dark matter $v_{p}$ is the same form as the speed distribution curve from visible light $v_{n}$. By using these three speed distribution curves it is possible to calculate with Equation (5) the speed distribution curve which corresponds to the speed distribution curve $V_{M}$ of four dimensional matter $M$. In the publication [1] has been calculated the halo speed distribution curve, which corresponds to the curve $V_{M}$ of Equation (10). They can be compared. The system of galaxy rotation of this study is a hypothesis, and it must be proved to be true or not true. With two pairs of values of rotational speeds $V_{M 1}$ and $v_{M 2}$ and radii $R_{1}$ and $R_{2}$ it can be done.

In the publication [1] has been presented rotational speed distributions curves of the galaxy NGC 3198. The speed distribution curve of galactic halo in that 


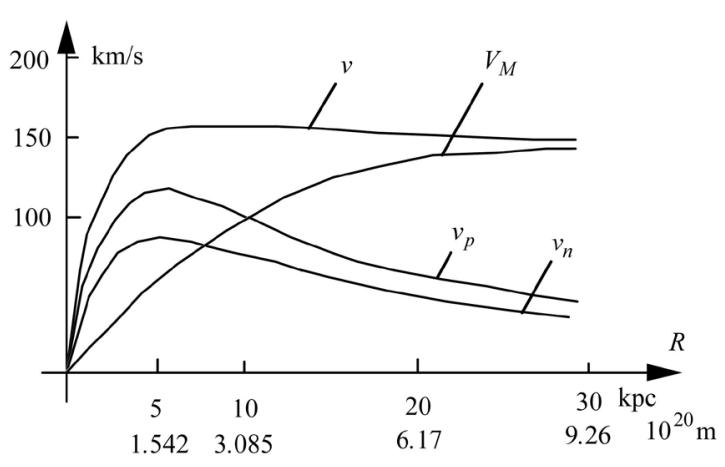

Figure 7. Total speed distribution curve $v$ and speed distribution curve of visible light $V_{n}$ which is the same shape as the speed distribution curve of ordinary dark matter $v_{p}$. Speed distribution curve $V_{M}$ of the four dimensional mass $M$ has been calculated with Equation (10), and the speed curves $V_{p}$ and $V_{n}$ have been calculated from the speed curve $v_{M}$ with Equation (5) using the fact that speed curve $v$ is level shape curve.

publication corresponds to the speed distribution curve $v_{M}$ of four dimensional mass of this study. In order to find out how well this four dimensional model functions, the speed distribution curve $v_{M}$ of four dimensional mass has been calculated by using these rotational radius and speed values.

$$
\begin{aligned}
& R_{1}=3 \times 10^{20} \mathrm{~m}, \quad R_{2}=8 \times 10^{20} \mathrm{~m} \\
& v_{M 1}=80 \mathrm{~km} / \mathrm{s}, \quad v_{M 2}=130 \mathrm{~km} / \mathrm{s}
\end{aligned}
$$

Distance of the four dimensional mass $M$ from the center of galaxy, Equation (15) and Equation (17)

$$
\begin{gathered}
a=\frac{v_{M 1} R_{2}}{v_{M 2} R_{1}}=\frac{0.8 \times 8}{1.3 \times 3}=1.64 \\
X^{\prime}=\sqrt{\frac{R_{2}^{2}-a R_{1}^{2}}{a-1}}=\sqrt{\frac{8^{2}-1.64 \times 3^{2}}{0.64}} \times 10^{20} \mathrm{~m}=8.8 \times 10^{20} \mathrm{~m}
\end{gathered}
$$

Four dimensional mass $M$, Equation (11)

$$
\begin{aligned}
M & =\frac{v_{M 1}^{2}\left(R_{1}^{2}+\left(X^{\prime}\right)^{2}\right)^{2}}{R_{1}^{2} \gamma} \\
& =\frac{0.8^{2} \times 10^{10}\left(3^{2} \times 10^{40}+8.8^{2} \times 10^{40}\right)^{2}}{3^{2} \times 10^{40} \times 6.67 \times 10^{-11}} \mathrm{~kg} \\
& =79.7 \times 10^{61} \mathrm{~kg}
\end{aligned}
$$

From Equation (10) the effect of four dimensional mass $M$ on the rotational speed distribution curve $V_{M}$ is obtained as follows:

$$
v_{M}=\sqrt{\frac{R^{2} \gamma M}{\left(R^{2}+\left(X^{\prime}\right)^{2}\right)^{2}}}=\sqrt{M} \cdot \sqrt{\frac{R^{2} \gamma}{\left(R^{2}+\left(X^{\prime}\right)^{2}\right)^{2}}}
$$

As it is obvious that the effects of three and fourth dimensional masses are the same, except for the effect of the distance $R$, it can be inferred that the coefficients of gravity are the same, except for the quality, $\gamma=6.67 \times 10^{-11} \mathrm{~N} \cdot \mathrm{m}^{3} \cdot \mathrm{kg}^{-2}$. 


\section{Conclusions}

The total rotational speed distribution of galaxy NGC 3198 has been presented in the publication [1] which corresponds to the total rotational speed $v$ in this study, and the rotational speed components of visible and ordinary dark matter which corresponds to the rotational speed $v_{n}+v_{p}$ in this study, and rotational speed component of galactic halo which corresponds in this study the rotational speed component $v_{M}$ of four dimensional matter $M$, Equation (10).

1) In order to find out how well this four dimensional model functions, the speed distribution curve $v_{M}$ of four dimensional mass $M$ has been calculated by using two pairs of rotational radius and speed values which are approximately the same as the speed curve values of galactic halo of the publication [1]. By that means the theoretical speed curve $v_{M}$ of four dimensional mass $M$ can be compared to the corresponding speed curve of real galaxy rotation. By using these values it was obtained the four dimensional mass value $M=79.7 \times 10^{61} \mathrm{~kg}$, Equation (11) and the distance above the center of galaxy in the four dimensional axis $X^{\prime}=8.8 \times 10^{20} \mathrm{~m}$, Equation (17). The rotational speed distribution component of the four dimensional matter $v_{M}$ was calculated from Equation (10), and it is presented in Figure 8. By comparing it with the speed distribution of halo in the publication [1], it was inferred that it corresponded quite good to the halo distribution. It gives confirmation, and probability increases that the four dimensional system of this study is right. The four dimensional matter was supposed to be at one point, and its distribution in the space is one source of inaccuracy.

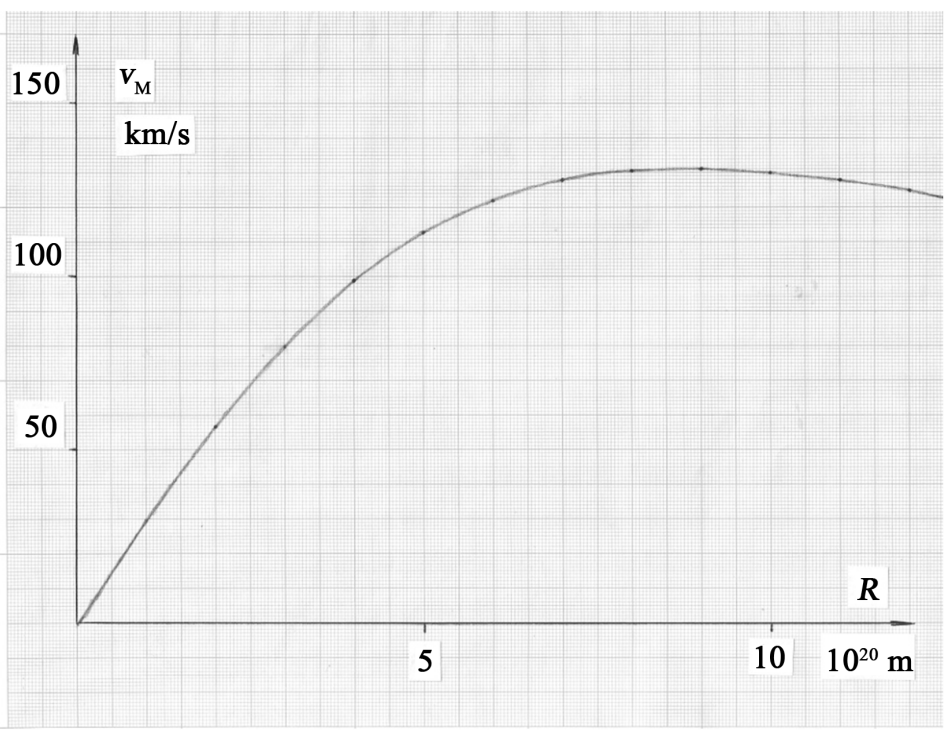

Figure 8. Rotational speed distribution $v_{M}$ of the four dimensional mass $M$ has been presented as function of radius $R$. Rotational speed distribution $V_{M}$ is calculated with Equation (10) using values of mass $M=79.7 \times 10^{61} \mathrm{~kg}$ and its distance from the center of galaxy $X^{\prime}=8.8 \times 10^{20} \mathrm{~m}$. The maximum value of the speed distribution $V_{M}=130 \mathrm{~km} / \mathrm{s}$ is at the radius $R=9.0 \times 10^{20} \mathrm{~m}(29.2 \mathrm{kpc})$, and it indicates the maximum radius of galaxy. The rotational speed distribution $V_{M}$ corresponds to the component of halo speed distribution of galaxy NGC 3198 in the publication [1]. 
The other source of inaccuracy is the redshift measurements and the evaluation of the mass distribution of ordinary dark matter. The halo speed distribution curve of the publication [1] corresponds quite well to the speed distribution of four dimensional mass $V_{M}$ until the radius $R=30 \mathrm{kpc}$. In the publication [1] speed measurements has been done until radius $R=30 \mathrm{kpc}$, which obviously is the maximum radius of galaxy, but however the halo curve continue until radius $R=50 \mathrm{kpc}$. In the region $30-50 \mathrm{kpc}$ the halo speed curve of the publication [1] and the four dimensional matter speed curve $V_{M}$ begins to be separated from each other significantly. The speed curve of four dimensional matter $v_{M}$ decreases in this region, but the halo speed curve of the publication [1] continues to increase. It is possible that the maximum radius of galaxy is determined according to the point in which the speed curve $v_{M}$ begins to decrease.

2) The generation process of galactic system may be explained like this: The force of gravitation of the four dimensional mass $M$ acts on the rotational plane of galaxy. It has no component at the direction of the fourth dimension because the three dimensional mass of galaxy of stars, planets, gas, dust and other matter have not gravity force of fourth dimension. The result is that the four dimensional mass $M$ generates a gravitational field which has a great hole at the center of galaxy. The gravitational field of the four dimensional mass accelerates three dimensional mass of stars, planets, dust, and other matter into the speed of rotation in which it rotates round about the center of galaxy. In this manner the hole in the gravitational field of the four dimensional mass $M$ fills up, and the typical constant speed outer boundary regions of galaxies have been generated. The gravity field of the four dimensional matter $M$ accelerates a star to the rotational speed somewhat above $130 \mathrm{~km} / \mathrm{s}$, in which case it retain rotating the galaxy in the border region, and if the star loses kinetic energy and speed, it begins to rotate the galaxy at the center region, and if the star accelerates considerably more than $130 \mathrm{~km} / \mathrm{s}$, then the gravity force of the four dimensional matter cannot hold it, and it moves out of the gravity field.

3) In Figure 9 the effect of the distance $X^{\prime}$ on the maximum radius of galaxy $R$ has been studied. The maximum radius of galaxy is at the point of arrow Figure 9. As the distance $X^{\prime}$ of the four dimensional mass $M$ increases from the center of galaxy, the maximum radius of the galaxy increases. By applying Equation (18) for more massive four dimensional mass concentrations, it can be obtained rotation curves of the publication [2]. The effect of the spherical galactic halo has been neglected, and it is supposed that the distribution of the mass of halo is such that it has no significant effect on the rotational speed distribution.

4) Justification: Fitting the four dimensional galaxy rotation system to the measured values of the galaxy rotation.

Point 1 . The speed distribution curve of four dimensional mass $V_{M}$ is a good fitting to the corresponding measured halo speed distribution curve of publication [1].

Point 2. The generation of the galaxy system can be inferred.

Point 3. The calculation of the speed distribution curve of four dimensional 


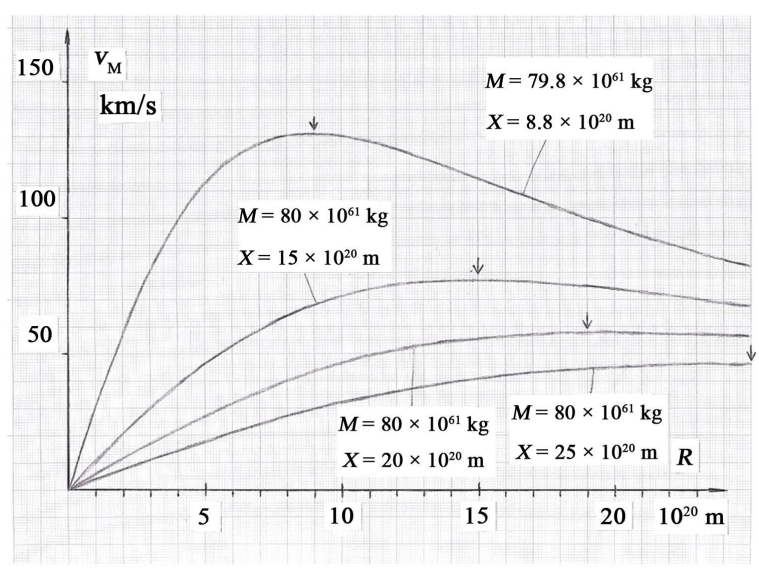

Figure 9. Four rotational speed distribution curves $V_{M}$ presented as function of radius $R$ from the center of galaxy. The uppermost speed distribution curve is from Figure 8, which is the speed distribution curve of the four dimensional mass $M$ which corresponds to the halo component of rotational speed distribution of galaxy NGC 3198. The three speed distribution curves under indicate the effect of the distance $X^{\prime}$ on the maximum radius of galaxy $R$, which is at the point of arrow. As the distance $X^{\prime}$ increases $X^{\prime}=8.8 \times 10^{20}$ $\mathrm{m} \rightarrow X^{\prime}=25 \times 10^{20} \mathrm{~m}(28.5 \mathrm{kpc} \rightarrow 81 \mathrm{kpc})$ the maximum radius of galaxy increases $R=9.0$ $\times 10^{20} \mathrm{~m} \rightarrow 25 \times 10^{20} \mathrm{~m}(29.2 \mathrm{kpc} \rightarrow 81 \mathrm{kpc})$, or even more because the two lowest speed distribution curves $V_{M}$ are almost horizontal at the point of arrow. The maximum speed value of the speed distribution curve $V_{M}$ (the point of arrow) is directly proportional to $\sqrt{M}$.

mass $V_{M}$ with different mass $M$ and distance $X^{\prime}$ values generate the measured speed distribution curves of galaxies in publication [2].

\section{Conflicts of Interest}

The author declares no conflicts of interest regarding the publication of this paper.

\section{References}

[1] Van Albada, T.S., Bahcall, J.N., Begeman, K. and Sanscisi, R. (1985) Distribution of Dark Matter in the Spiral Galaxy NGC 3198. The Astrophysical Journal, 295, 305313. https://doi.org/10.1086/163375

[2] Rubin, V.C. (1983) The Rotation of Spiral Galaxies. Science, 220, 1339-1344. https://doi.org/10.1126/science.220.4604.1339

[3] Ostriker, J.P., Peebles, P.J.E. and Yahil, A. (1974) The Size and Mass of Galaxies, and the Mass of the Universe. The Astrophysical Journal, 193, L1-L4. https://doi.org/10.1086/181617

[4] Burrows, A. and Liebert, J. (1995) Probing Dark Matter. Nature, 373, 191-192. https://doi.org/10.1038/373191a0

[5] Moster, B.P., Somerville, R.S., Maulbetsch, C., van den Bosch, F.C., Maccio, A.V., Naab, T. and Oser, L. (2010) Constraints on the Relationship between Stellar Mass and Halo Mass at Low and High Redshifts. The Astrophysical Journal, 710. https://doi.org/10.1088/0004-637X/710/2/903

[6] Behroozi, P.S., Wechsler, R.H. and Wu, H. (2012) The Rockstar Phase-Space Temporal Halo Finder and the Velocity Offsets of Cluster Cores. The Astrophysical Jour- 
nal, 762. https://doi.org/10.1088/0004-637X/762/2/109

[7] Panek, R. (2020) A Cosmic Crisis. Scientific American.

[8] Roos, M. (2003) Introduction to Cosmology. 3rd Edition, John Wiley \& Sons Ltd.

\section{List of Variables}

Dimensions of ordinary space, $x, y, z$

Fourth distance dimension, $x^{\prime}$

Four dimensional mass, $M$

Ordinary visible galactic mass, $M(x)$

Location of the mass $M$ on the fourth distance dimension, $X^{\prime}$

Rotational speed distribution curve, $V$

Rotational speed distribution component of the mass $M, V_{M}$

Rotational speed distribution component of visible mass, $V_{n}$

Rotational speed distribution component of ordinary dark matter, $V_{p}$

Radius of rotation of the galactic mass, $R$

Mass of a star, $m$ 